\title{
Generation of terahertz pulses from the island films of topological insulator $\mathrm{Bi}_{2-\mathrm{x}} \mathrm{Sb}_{\mathrm{x}} \mathrm{Te}_{3-\mathrm{y}} \mathrm{Se}_{\mathrm{y}}$
}

\author{
$\underline{\text { K.A. Kuznetsov }}^{1}$, G.Kh. Kitaeva ${ }^{1}$, P.I. Kuznetsov ${ }^{2}$, and G.G. Yakushcheva ${ }^{2}$ \\ ${ }^{1}$ M.V. Lomonosov Moscow State University, 119991 Moscow Russia, kirill-spdc@yandex.ru \\ ${ }^{2}$ Kotelnikov IRE RAS (Fryazino branch), 141190 Fryazino, Russia
}

A great interest to topological matter is stimulated by the study of the $\mathrm{THz}$ response to laser radiation. Angle-resolved photoemission spectroscopy (ARPES) indicates the presence of Dirac electrons in many topological insulators (TI) [1] Besides of fundamental aspects, TI's are very perspective objects for practical purposes both $\mathrm{THz}$ detectors as $\mathrm{THz}$ emission sources.

In this work, we perform a study of the $\mathrm{THz}$ emission properties of $\mathrm{Bi}_{2-\mathrm{x}} \mathrm{Sb}_{\mathrm{x}} \mathrm{Te}_{3-\mathrm{y}} \mathrm{Se}_{\mathrm{y}}$ thick films with thickness of hundreds $\mathrm{nm}$ and one island film with total thickness about $40 \mathrm{~nm}$ grown by MOCVD method on sapphire substrate. Rhombohedral $\mathrm{Bi}_{2}$ ${ }_{x} \mathrm{Sb}_{\mathrm{x}} \mathrm{Te}_{3-\mathrm{y}} \mathrm{Se}_{\mathrm{y}}$ films were grown on (0001) Al2O3 substrates with thin $(10 \mathrm{~nm})$ ZnTe buffer layer with orientation (111) at atmospheric pressure of hydrogen in horizontal quartz reactor. An example of AFM scan of thin island film surface is shown in Fig. 1.

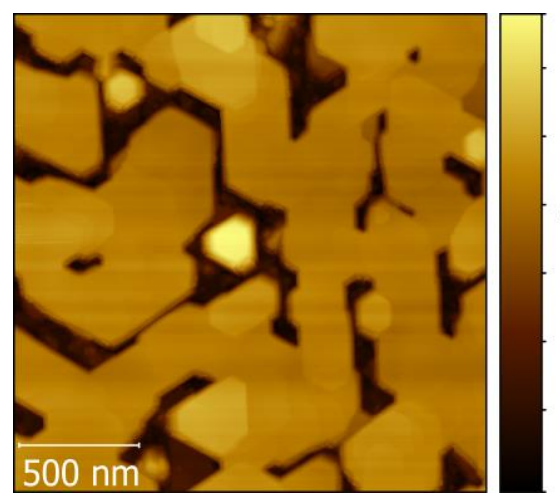

$69.9 \mathrm{~nm}$

60.0

50.0

40.0

30.0

20.0

10.0

0.0

Fig. 1. AFM scan of the TI island film's surface

We use experimental setup for the terahertz emission time-domain spectroscopy (TDS) in the backward geometry. The pump is realized by the Erbium fiber laser in the Q-switched mode locking near a threshold regime at $1.56 \mu \mathrm{mm}$ wavelength. $\mathrm{Er}^{+}$-laser laser operated in the picosecond mode, generating optical pulses of 2.5 ps duration with the repetition rate of $70 \mathrm{MHz}$. The pump bam was divided into two unequal parts with a mean power of 100 and $20 \mathrm{~mW}$, respectively. The low-power beam was focused on a photoconductive dipole antenna. The high-power beam, after propagation through the delay line and an optical chopper, was focused by the through a hole in the parabolic mirror onto the TI sample. The angle of incidence was $15^{\circ}$. The sample was in contact with copper electrodes (the distance between them was 0.5 $\mathrm{mm}$ ) so that external voltage could be applied. The $\mathrm{THz}$ radiation generated in the sample in the backward direction was collected by four parabolic mirrors and focused on the commercial $\mathrm{THz}$ antenna. The antenna was oriented to register vertical components of the $\mathrm{THz}$ field collinear with the polarization of the pump. Thus, the electric field of $\mathrm{THz}$ waves generated in the direction opposite to pumping was measured at different times after the arrival of the pump pulse.

We have shown that the intensity of the $\mathrm{THz}$ signal from the island film is 25 times greater than that in "thick" TI samples. In order to demonstrate the amplification effect for $\mathrm{THz}$ radiation using an external electric field, we applied a voltage between the electrodes in contact with a surface of the island film. Fig. 2 shows the waveforms of $\mathrm{THz}$ radiation from an island film with a bias voltage. When the polarity of the bias voltage changed, the $\mathrm{THz}$ signal also changed its phase by 180 degrees. Thus, for the first time, the mode of the radiating antenna in a topological insulator was demonstrated.

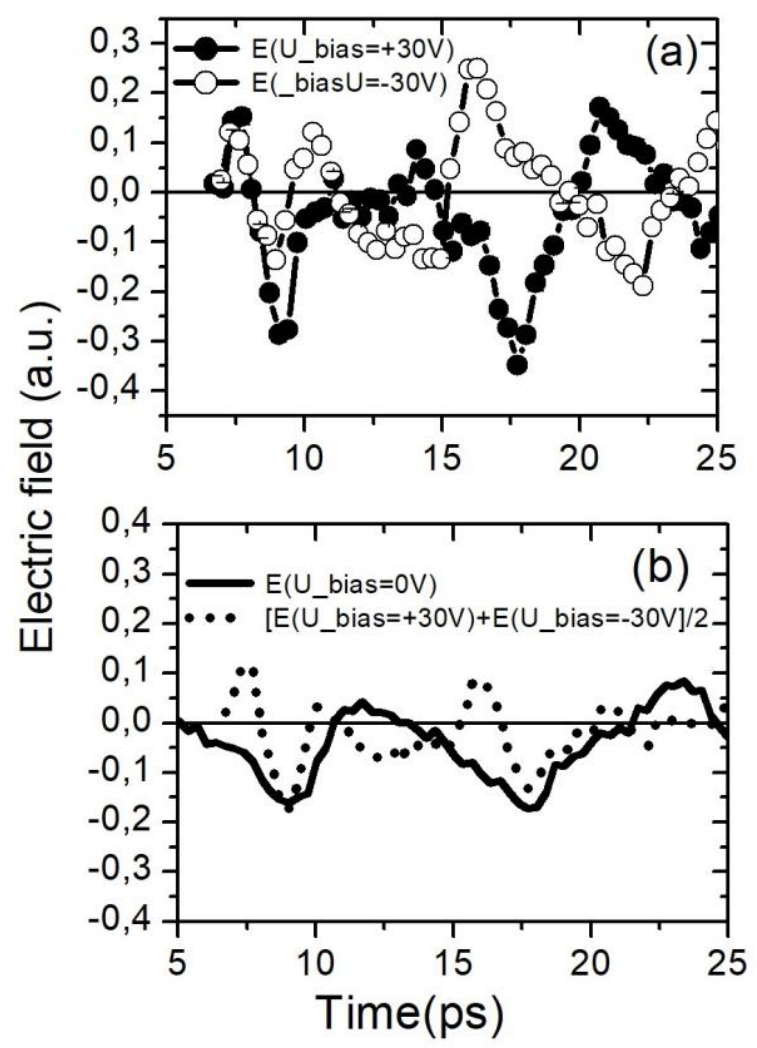

Fig. 2. Waveforms of the $\mathrm{THz}$ pulses

The observed waveforms contain some additional signal, which does not change its polarity with the voltage. To reveal this part we have summarized the signals, observed with $+30 \mathrm{~V}$ and $-30 \mathrm{~V}$ voltages. Half of this sum is shown in Fig.2b (see the dotted line). The opposite-sign parts of the signals from Fig.2a almost canceled each other, so that one could expect 
to observe the residual constant part of the same shape as the zero-voltage signal. However, this is not true. For comparison, we repeat the zero-voltage signal together with this residual signal. One can see that the antenna pulses contain additional parts corresponding to higher modulation frequencies. This fact is illustrated in Fig. 3 where the spectra of the both waveforms are shown.

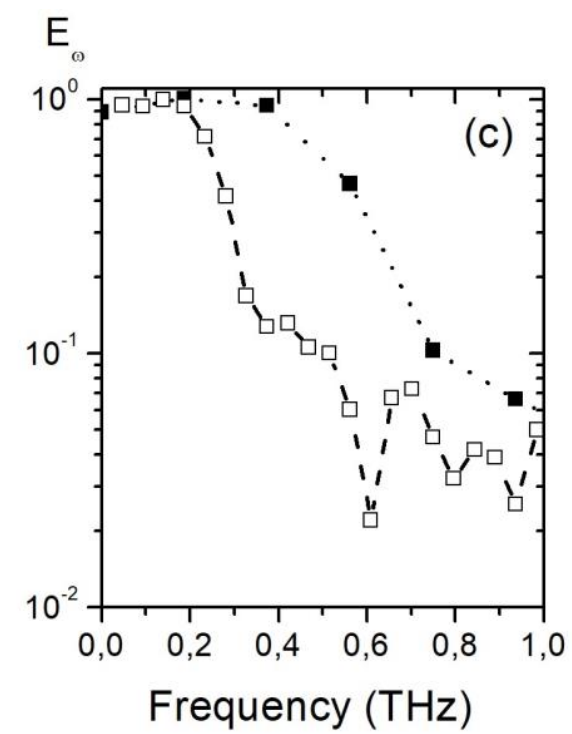

Fig. 3 Spectrum of zero-voltage signal (hollow squares) and spectrum of half-sum of signals at external bias (filled squares).
The spectrum of the residual part of the antenna signal is about two-times broader than the spectra of all the other signals, i.e. the zero-voltage signal, and the polarity-sensitive parts of $+30 \mathrm{~V}$ and $-30 \mathrm{~V}$ voltages signals. The peak frequency corresponds to times that are of order or even less than the pump pulse duration. It means that some carrier relaxation effects proceed at considerably shorter relaxation times, than it is usually assumed to describe the $\mathrm{THz}$ emission of free TI samples. We believe that this effect arises from the relaxation of fast non-equilibrium charge carriers accelerated by an external field. Probably electrons are captured by the traps that are p-type impurities with energy above the bulk valence band maximum in the energy of 3-6 meV or other defects.

The authors are grateful to Dr. T.V. Murzina, for discussions and help. The study of $\mathrm{THz}$ emission was performed under the support of the RFBR grant № 16-02-258a. The growth and characterization of the $\mathrm{Bi}_{2-\mathrm{x}} \mathrm{Sb}_{\mathrm{x}} \mathrm{Te}_{3-\mathrm{e}} \mathrm{Se}_{\mathrm{y}}$ samples was supported by grant 17 19-01057 of the Russian Science Foundation.

\section{References}

1. M. Z. Hasan and C. L. Kan. Colloquium: Topological insulators // Rev. Mod. Phys. 2010, V.82,P. 3045. 